\title{
A Beginners Guide to: Atom-Probe Tomography
}

\author{
E.A. Marquis* \\ * Department of Materials Science and Engineering, University of Michigan, Ann Arbor MI 48109
}

Among the available imaging and spectroscopy technique, atom-probe tomography (APT) is unique in its ability to image and analyze single atoms with theoretically equal sensitivity to all elements of the periodic table. As such, APT is quickly becoming a useful, if not required, technique to analyze nanoscale features in materials. Examples of APT applications are numerous. As illustrated in Figure 1, clustering mechanisms in light alloys [1], grain boundary segregation in various alloys [2,3], quantum structures in semiconductor materials [4], oxide microstructures [5,6], analysis of dopants in Si-based devices [7] are just a few successful stories where APT has provided invaluable insights into the structure of materials.

This tutorial will be designed for anybody interested in learning about APT with no particular required background. Basic concepts, specimen preparation, data reconstruction and analysis, and applications to materials studies will be introduced.

APT provides atom-by-atom three-dimensional reconstructions of small volumes of materials, typically $80 \mathrm{~nm} \times 80 \mathrm{~nm} \times$ few hundreds $\mathrm{nm}$, by combining field ionization, time of flight mass spectrometry and position sensitive detection. Specimens in the form of sharp needles with radius of curvature at the apex less than $\sim 100 \mathrm{~nm}$ are required. Voltages (typically $2-14 \mathrm{kV}$ ) are applied on the specimens create high electric fields just below the evaporation fields of the surface atoms (2$40 \mathrm{~V} / \mathrm{nm}$ depending on the material and microstructure). Application of short voltage pulses or laser pulses is used to trigger the evaporation process and determine the time of departure of ions from the specimen surface. Ion detection and positioning is currently achieved by a combination of a delay line detector combined with micro-channel plates. Three dimensional data reconstruction is then obtained assuming a simple projection geometry of the tip surface onto the detector.

Examples of applications will cover specimen preparation that is usually performed by electropolishing or focused ion beam milling, reconstruction of data, data quantification using various examples taken from current materials science problems.

References

[1] S.P. Ringer, T. Sakurai, I.J.Polmear, Acta Mater. 45 (1997) 3731.

[2] E.A. Marquis, R. Hu, T. Rousseau, Journal of Nuclear Materials (2011) In press

[3] E.A. Marquis, M.K. Miller, D. Blavette, S.P. Ringer, C.K. Sudbrack, G.D.W. Smith. MRS Bulletin 34 (2009) 72

[4] B.P. Gorman, A.G. Norman, Y. Yan. Microscopy and Microanalysis 13 (2007) 493.

[5] Y.M. Chen, T. Ohkubo, M. Kodzuka, K. Morita, K. Hono K. Scripta Materialia 61 (2009) 693

[6] E.A. Marquis, N.A. Yahya, D.J. Larson, M.K. Miller, R.I. Todd. Materials Today 13 (2010) 42

[7] L.J. Lauhon, P. Adusumilli, P. Ronsheim, P.L. Flaitz, D. Lawrence. MRS Bulletin 34 (2009) 72

This tutorial will be recorded via a lecture capture system and made available to MAS and MSA members at no charge on the MSA website (hhtp:// www.microscopy.org) 
(A)

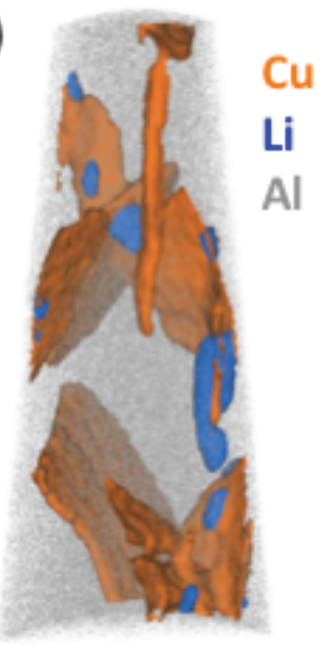

(C)

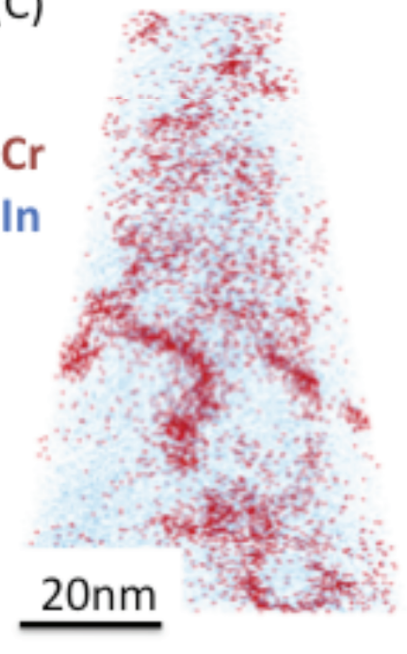

(B)

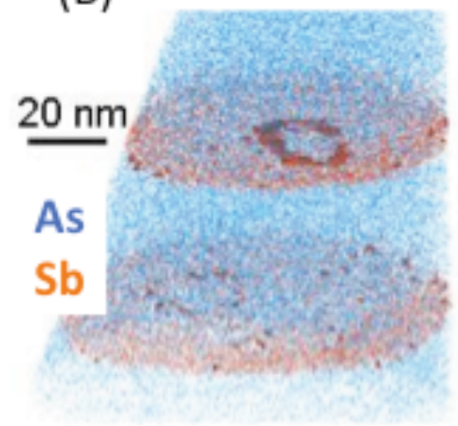

(D)

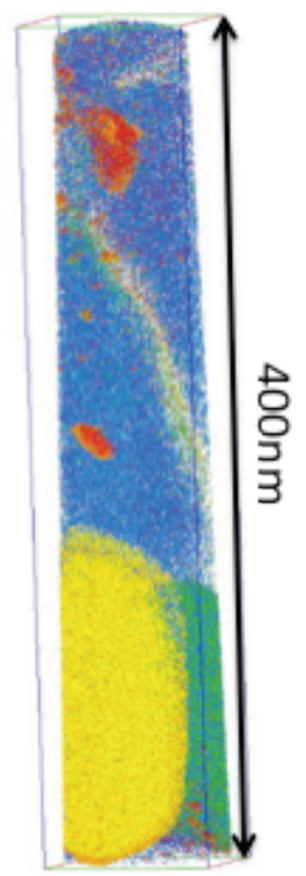

FIG. 1. 3D reconstructions of (A) Cu-rich aand Li-rich precipitates in an $\mathrm{Al}$ alloy, (B) quantum rings in $\mathrm{GaAs} / \mathrm{GaSb}$ structures. (C) Cr distribution in $\mathrm{Cr}$-doped indium oxide and (D) Cr-depleted grain boundaries and second phases in an oxide dispersion strengthened steel. 